\title{
Short-term impact of COVID-19 on quality of life, perceived stress, and serious psychological distress in an adult population in the midwest United States
}

\author{
Leonard E. Egede ${ }^{1,2}$ (1) $\cdot$ Rebekah J. Walker ${ }^{1,2} \cdot$ Aprill Z. Dawson $^{1,2} \cdot$ Amy Zosel $^{4} \cdot$ Sanjay Bhandari ${ }^{1,2}$. \\ Sneha Nagavally ${ }^{1,2} \cdot \operatorname{Ian}$ Martin $^{3} \cdot$ Michael Frank $^{4}$
}

Accepted: 5 January 2022 / Published online: 12 January 2022

(c) The Author(s), under exclusive licence to Springer Nature Switzerland AG 2022

\begin{abstract}
Purpose This study aimed to investigate changes over time in quality of life, perceived stress, and serious psychological distress for individuals diagnosed with COVID-19 in an urban academic health system.

Methods Phone-based surveys were completed with adult patients tested for COVID-19 during emergency department visits, hospitalizations, or outpatient visits at the Froedtert and Medical College of Wisconsin Health Network. Data were then matched to medical record data. Unadjusted and adjusted mixed effects linear models using random intercept were run for each outcome (physical health-related quality of life, mental health-related quality of life, perceived stress, and serious psychological distress) with time (baseline vs 3-month follow-up) as the primary independent variable. Individuals were treated as a random effect, with all covariates (age, sex, race/ethnicity, payor, comorbidity count, hospitalization, and intensive care unit (ICU) stay) treated as fixed effects.

Results 264 adults tested positive for COVID-19 and completed baseline and 3-month follow-up assessments. Of that number, $31.8 \%$ were hospitalized due to COVID-19, and $10.2 \%$ were admitted for any reason to the ICU. After adjustment, patients reported higher physical health-related quality of life at 3 months compared to baseline $(0.63,95 \% \mathrm{CI} 0.15,1.11)$ and decreased stress at 3 months compared to baseline $(-0.85,95 \% \mathrm{CI}-1.33,-0.37)$. There were no associations between survey time and mental health-related quality of life or serious psychological distress.

Conclusions Results suggest the influence of COVID-19 on physical health-related quality of life and stress may resolve over time, however, the influence of mental health on daily activities, work, and social activities may not.
\end{abstract}

Keywords COVID-19 $\cdot$ Mental health $\cdot$ Stress $\cdot$ Serious psychological distress $\cdot$ Quality of life

\section{Introduction}

Leonard E. Egede

legede@mcw.edu

1 Division of General Internal Medicine, Department of Medicine, Medical College of Wisconsin, 8701 Watertown Plank Rd., Milwaukee, WI 53226, USA

2 Center for Advancing Population Science, Medical College of Wisconsin, 8701 Watertown Plank Rd, Milwaukee, WI 53226, USA

3 Department of Emergency Medicine, Medical College of Wisconsin, 8701 Watertown Plank Rd., Milwaukee, WI 53226, USA

4 Division of Infectious Disease, Department of Medicine, Medical College of Wisconsin, 8701 Watertown Plank Rd., Milwaukee, WI 53226, USA
Coronavirus disease 2019 (COVID-19), caused by severe acute respiratory syndrome coronavirus 2 (SARS-CoV-2), was declared a public health emergency of international concern by the World Health Organization (WHO) on January 30, 2020 and has taken an unprecedented toll on the public health and medical communities around the world [1]. Significant disparities have been noted in the United States, for example, in Milwaukee County, Wisconsin, 22\% of confirmed cases and $28 \%$ of deaths have been in African Americans [2,3]. Though nearly $98 \%$ of those diagnosed with COVID-19 recover, little is known about the clinical course of these patients, clinically and in terms of quality of life [1]. 
Long-term effects of COVID-19 are increasingly recognized and described in the literature, using terms such as, "Long COVID," "Post-acute COVID-19 syndrome," and "Post-Acute Sequelae of SARS-CoV-2 Infection (PASC)" [4]. Based on a systematic review and meta-analysis, the syndrome has been found to include up to 55 different longterm effects [4]. Both hospitalized and non-hospitalized patients may report multiple symptoms 3 months after onset of COVID-19 [5]. However, a recent manuscript noted problems related to lack of agreed upon definitions of postCOVID symptoms, which include both ongoing symptomatic COVID-19 (from 4 to 12 weeks) and post-COVID-19 syndrome (symptoms greater than 12 weeks) [6]. In a study of 143 patients, who had been hospitalized and recovered from COVID-19, 87.4\% reported persistence of at least 1 symptom at 60-day follow-up, most commonly fatigue and dyspnea [7]. Symptoms lasting greater than or equal to 28 days, including fatigue, headache, dyspnea, and anosmia), highlight the potential to significantly impact the quality of life in patients [8]. In a study conducted in Norway, lower scores were found in a number of quality of life domains for individuals that experienced COVID-19 compared to the general population, including mobility, usual activities, and energy/fatigue [9].

In addition to persistent physical symptoms, perceived stress, and psychological sequelae are a mounting concern. A large retrospective study of 236,379 survivors of COVID19 found substantial neurological and psychiatric morbidity in the 6 months after COVID-19 infection, with the risks greatest in patients who had severe COVID-19 [10]. These psychological sequelae may increase the demand for mental health care during and after the COVID-19 pandemic, however, the World Health Organization reported that the COVID-19 pandemic has disrupted or halted critical mental health services in $93 \%$ of countries worldwide [11]. The mental health-related impact of the pandemic is not limited to those diagnosed with COVID-19. For example, a study of pandemic-related stressors and overall psychological distress found that nearly half of participants noted an increase in mild psychological distress regardless of COVID-19 infection, with distress prior to the pandemic, financial stressors, and work/life balance stressors all associated with higher distress during the pandemic [12]. However, evidence suggests that there is a prolonged mental health impact, including perceived stress, on individuals diagnosed with COVID19 compared to healthy controls, with hypotheses that it is most pronounced 1-4 months after diagnosis [13].

Given the limited information that exists regarding the change in physical and mental health-related quality of life over time following COVID-19 diagnosis, the objective of this study was to investigate changes from baseline to 3-month follow-up in quality of life, perceived stress, and serious psychological distress for individuals diagnosed with
COVID-19 in an academic health system in Southeast Wisconsin. The urban, Midwest setting, and racially/ethnically diverse populations provide important information on longterm impacts of COVID-19 on adults in the US.

\section{Study data and methods}

\section{Population}

Adults aged 18 and older, who were tested for COVID-19 in the Froedtert and Medical College of Wisconsin Health Network were eligible for participation in the study. The Froedtert and Medical College of Wisconsin Health Network comprised five hospitals and nearly 40 health centers and clinics and is located in southeastern Wisconsin. The primary academic medical center and hospital is located in Milwaukee County, a racially and ethnically diverse county with sociodemographic make-up similar to the US population.

Phone-based surveys were completed with patients who had emergency department visits at which a COVID-19 test was administered, hospitalizations as patients under investigation (PUIs) or with a diagnosis of COVID-19, and adults tested for COVID-19 in outpatient settings. Initial response to participation in the study was high, with $32 \%$ of individuals contacted agreeing to participate. Only 11 participants declined participation after asking for additional information on the study and beginning the consent process. Data were not collected on individuals who indicated they were not interested in participation or did not complete the consent. Individuals who were hospitalized were contacted while in the hospital or within weeks of discharge, and those not hospitalized were contacted following indication in the medical record of test results. On average, participation in the study occurred 46 days after a positive COVID-19 test result (mean 45.8, standard deviation 32.7).

All recruitment were conducted over telephone with enrollment occurring from April 21, 2020 through September 14, 2020. Verbal informed consent was obtained at baseline, with questionnaires completed at baseline and 3-month follow-up. The drop-out rate between baseline and 3-month follow-up was low, with only 43 participants (14\% of the initial 307 completing a baseline survey) not completing the 3-month follow-up survey. Research staff contacted participants to complete missing data following internal audits of surveys to ensure all scales could be scored, resulting in no missing data for participants who completed both baseline and 3-month survey follow-up. Survey data were then matched to medical record data from the Froedtert and Medical College of Wisconsin Epic ${ }^{\circledR}$ Electronic Health Record System. All study procedures were reviewed and approved by the Medical College of Wisconsin Institutional Review Board prior to initiation of enrollment. 


\section{COVID-19 status}

COVID-19 positive status was determined based on testing completed following two nasopharyngeal specimen and oropharyngeal specimen swabs. Swabs were transported to the laboratory using viral transport media and testing followed Centers for Disease Control and Prevention (CDC) COVID-19 assay with RNA extracted using the BioMerieux and real-time polymerase chain reaction using ABI 7500 Fast Dx thermal cyclers. This study includes data from the 345 participants who completed both baseline and 3-month surveys and who tested positive for COVID-19.

\section{Outcome measures}

Phone-based surveys conducted either while patients were in the hospital or after discharge captured patient-reported outcomes and social determinant factors at baseline and 3 months later. Baseline was set at date of first survey, which was conducted by a research assistant who read all questions and answers to the participant and noted responses. Participants were not contacted within the ICU and gave verbal consent after indicating both interest and ability to participate. Data included the three primary outcomes for this study.

(1) Quality of life physical and mental health-related quality of life measured using the SF-12 v1 scale [14]. This 12 -item scale is a valid and reliable instrument of functional status and is widely used to provide summary physical health-related quality of life (PCS-12) and mental health-related quality of life (MCS-12) scores across populations [14]. The SF-12 reproduces $90 \%$ of the variance, and scores are considered interchangeable with those from the longer SF-36 scale [14]. The testretest reliability for PCS is 0.89 and 0.76 for MCS [14]. Scores were calculated using norm-based methods for PCS-12 and MCS-12 as recommended by Ware et al. [14]. Scores for each of the MCS and PCS range from 0 to 100 , with higher scores indicating better mental and physical health-related quality of life, respectively [15]. In this sample, the Cronbach's alpha for the PCS score was 0.60 and for the MCW score was 0.60.

(2) Serious psychological distress (SPD) measured using the K6 scale [16]. This 6-item scale was originally developed for population-level screening of non-specific psychological distress and allows discrimination between DSM-IV cases and non-cases [16]. It has good precision and consistent psychometric properties across populations, being routinely used by the US National Health Interview Survey. The Cronbach's alpha for the $\mathrm{K} 6$ is 0.86 indicating very good reliability [17]. The K6 scale ranges from 0 to 24 with higher scores indicating higher levels of distress [16]. In this sample, the Cronbach's alpha for the K6 scale was 0.81 .

(3) Perceived stress measured using the perceived stress scale [18]. This 4-item scale assesses the degree to which a respondent finds situations stressful by asking about the frequency of feelings related to events in the prior month [18]. Cronbach alpha scores of 0.69 show reliability and prior studies indicate the scale is correlated with stress, depression, and anxiety. Scoring for the Perceived Stress Scale was calculated by indicating never as ' 0 ,' almost never as ' 1 ,' sometimes as ' 2 ,' fairly often as ' 3 ,' and very often as ' 4 ' for questions "In the last month, how often have you felt that you were unable to control the important things in your life?" and "In the last month, how often have you felt difficulties were piling up so high that you could not overcome them?". Reverse coding was used for questions "In the last month, how often have you felt confident about your ability to handle your personal problems?" and "In the last month, how often have you felt that things were going your way?" with never as '4,' almost never as ' 3 ,' sometimes as ' 2 ' and fairly often as ' 1 ' and very often as ' 0 ' [18]. Scores for the perceived stress scale ranged from 0 to 16 , with higher scores indicating higher levels of perceived stress [18]. In this sample, the Cronbach's alpha for the perceived stress scale was 0.75 .

\section{Covariates}

Upon completion of baseline and 3-month follow-up surveys, data were matched using patient identifiers to extract medical record components that include COVID-19 test results for participants along with their demographics information, such as participant's age at the time of baseline survey, sex, race, participant's county, language, need for interpreter, and payor for health services. Comorbidities at the time of baseline for each individual were also collected using International Classification of Diseases (ICD-10) codes matched to the Elixhauser comorbidity categories [19]. A count of the comorbidities was created to capture the burden of comorbidities at baseline. Finally, information within the medical record was used to identify whether each individual was hospitalized and whether they had an ICU stay within 1 week following his/her COVID-19 test for any cause. To confirm the timeframe used to define hospitalization did not influence results, a sensitivity analysis was run defining hospitalization in the medical records as any time after the COVID-19 test, and hospitalization within one week after the COVID-19 test. Changing hospitalization timing did not change direction or strength of results.

Therefore, covariates included in the models were age (continuous), sex (male vs female), race/ethnicity 
(Non-Hispanic White, Non-Hispanic Black, Hispanic, and Other Race), payor (managed care/commercial, Medicaid, Medicare, self-pay, and other), comorbidity count (continuous), hospitalization (yes/ no), and ICU stay (yes/no).

\section{Statistical analysis}

Descriptive statistics were performed to summarize sample characteristics for the population with COVID-19-positive results. A series of regression models were then run to understand the independent association of time on each outcome. Outcomes were treated separately since each is a distinct construct with a validated scale used for measurement. Unadjusted models were run first to investigate the relationship without accounting for confounding. This allowed the comparison of estimates before and after adjustment. Mixed effects models were used to allow consideration of repeated measures and incorporate a random effect for individuals in the model. Regression modeling was chosen to allow investigation of the relationship after accounting for confounding variables and provide information on the independent association of time on each outcome. Least mean squares were run following mixed effects linear models to provide the adjusted means for ach outcome at baseline and 3-month follow-up.

Mixed effects linear models using a random intercept were run separately for eac of the four outcomes: first, physical health-related quality of life (PCS); second, mental health-related quality of life (MCS); third, Serious Psychological Distress (SPD); and fourth, Perceived Stress. Time (baseline vs 3-month follow-up) served as the primary independent variable. Individuals were treated as a random effect, with all covariates (age, sex, race/ethnicity, payor, comorbidity count, hospitalization, and ICU stay) treated as fixed effects. MS-SQL Server Management Studio version 2017 (SSMS) tool was used to fetch data from Clarity SQL server. Complete analysis was performed using $\mathrm{R}$ version-4.0.3, and the lme4 package from R's Comprehensive R Archive Network (CRAN) repository was used to run the linear mixed effects models [20]. This package provides functions for analyzing a range of mixed models, and the models and their components are represented using S4 classes and methods. The core computational algorithms are implemented using the 'Eigen' and 'RcppEigen' $\mathrm{C}++$ library for numerical linear algebra. All statistical model results were assessed for statistical significance using $p<0.05$.

\section{Results}

Sample characteristics for adults who tested positive for COVID-19 and completed both baseline and 3-month follow-up assessments are shown in Table 1 . There were 264
Table 1 Sample characteristics for participants who tested positive for COVID-19 completed baseline and a 3-month assessment $(n=264)$

Mean (SD) or $n \quad$ Mean (SD) at

(\%) at baseline 3-month followup

\begin{tabular}{|c|c|c|}
\hline \multicolumn{3}{|l|}{ Age } \\
\hline Mean (SD) & $49.2(16.7)$ & \\
\hline \multicolumn{3}{|l|}{ Gender } \\
\hline Female & $157(59.5 \%)$ & \\
\hline Male & $107(40.5 \%)$ & \\
\hline \multicolumn{3}{|l|}{ Race } \\
\hline Non-Hispanic White & $163(62.5 \%)$ & \\
\hline Non-Hispanic Black & $67(25.7 \%)$ & \\
\hline Hispanic & $15(5.7 \%)$ & \\
\hline Other/unknown & $16(6.1 \%)$ & \\
\hline \multicolumn{3}{|l|}{ Participant county } \\
\hline Other WI county/out of state & $9(3.4 \%)$ & \\
\hline Milwaukee county & $158(60.5 \%)$ & \\
\hline Waukesha county & $54(20.7 \%)$ & \\
\hline Washington/Ozaukee county & $25(9.6 \%)$ & \\
\hline Racine/Kenosha county & $15(5.7 \%)$ & \\
\hline \multicolumn{3}{|l|}{ Participant language } \\
\hline English & $251(96.5 \%)$ & \\
\hline Spanish & $6(2.3 \%)$ & \\
\hline Other/unknown & $3(1.2 \%)$ & \\
\hline \multicolumn{3}{|l|}{ Interpreter need } \\
\hline No & $253(98.1 \%)$ & \\
\hline Yes & $5(1.9 \%)$ & \\
\hline \multicolumn{3}{|l|}{ Payor } \\
\hline Managed care/commercial & $145(55.1 \%)$ & \\
\hline Medicaid & $32(12.2 \%)$ & \\
\hline Medicare & $69(26.2 \%)$ & \\
\hline Self-pay & $15(5.7 \%)$ & \\
\hline Other & $2(0.8 \%)$ & \\
\hline \multicolumn{3}{|l|}{ Comorbidity count } \\
\hline Mean (SD) & $1.4(1.89)$ & \\
\hline \multicolumn{3}{|l|}{ Hospitalization } \\
\hline No & $180(68.2 \%)$ & \\
\hline Yes & $84(31.8 \%)$ & \\
\hline \multicolumn{3}{|l|}{ ICU stay } \\
\hline No & $237(89.8 \%)$ & \\
\hline Yes & $27(10.2 \%)$ & \\
\hline $\begin{array}{l}\text { Physical health-related quality } \\
\text { of life }\end{array}$ & $52.7(4.9)$ & $53.3(4.6)$ \\
\hline $\begin{array}{l}\text { Mental health-related quality } \\
\text { of life }\end{array}$ & $61.8(1.5)$ & $61.6(1.4)$ \\
\hline Perceived stress scale & $4.5(4.1)$ & $3.6(3.9)$ \\
\hline Serious psychological distress & $10.8(10.4)$ & $10.4(4.2)$ \\
\hline
\end{tabular}


adults who tested positive for COVID-19 and completed baseline and 3-month follow-up assessments. Of that number, $31.8 \%$ were hospitalized due to COVID-19, and $10.2 \%$ were admitted for any reason to the ICU within 1 week following their COVID-19 test. The majority were female $(59.5 \%)$, non-Hispanic White $(62.5 \%)$, and had private insurance $(55.1 \%)$. The mean age was 49.2 years, and mean comorbidity count was 1.4. Mean physical health-related quality of life (PCS) scores were 52.7 (SD 4.9) at baseline, which is close to the US norm of 50, while mean mental health-related quality of- life (MCS) scores were 61.8 (SD $1.5)$ at baseline, which is higher than the US norm of 50. Means for baseline and 3 months were statistically significantly different for PCS ( $p$ value: 0.01 ; Cohen's $D: 0.16$ ) and perceived stress ( $p$ value: $<0.001$; Cohen's $D$ : 0.23 ). The means for baseline and 3 months were not significantly different for MCS ( $p$ value: 0.08 ; Cohen's $D: 0.11$ ) or SPD ( $p$ value: 0.08 ; Cohen's $D: 0.11$ ).

Table 2 shows results from unadjusted and adjusted linear regression models for the association between survey time (baseline vs 3 months) and quality of life (MCS and PCS). Unstandardized betas are reported along with the 95\% confidence interval (CI) and respective $p$ values. In the unadjusted model for PCS, patients at 3 months had a significantly higher physical health-related quality of life compared to baseline $(b$ : $0.62 ; 95 \%$ CI $0.15,1.09 ; p$ value: 0.01 ). This relationship held after adjusting for age, gender, race/ethnicity, payor, comorbidity count, hospitalization, and ICU stay ( $b$ : $0.63 ; 95 \%$ CI $0.15,1.11 ; p$ value: 0.01$)$. There was no association between survey time and MCS among patients who tested positive for COVID-19. Patient characteristics such as female sex and higher comorbidity count were associated with lower physical health-related quality of life, while female sex and older age were associated with higher mental health-related quality of life. Individuals who were hospitalized or had an ICU stay also had significantly lower physical health-related quality of life and significantly higher mental health-related quality of life.

Table 3 shows results of unadjusted and adjusted linear regression models for the association between survey time (baseline vs 3 months) and two additional outcomes, serious psychological distress (SPD) and perceived stress. There was no association between survey time and SPD among patients who tested positive for COVID-19. However, the relationship between survey time and perceived stress was statistically significant with patients at 3 months reporting lower stress $(b$ : $-0.91 ; 95 \%$ CI $-1.39,-0.43 ; p$ value: $<0.001)$ compared to baseline. This association was maintained in the fully adjusted model $(b:-0.85 ; 95 \% \mathrm{CI}-1.33,-0.37$; $p$ value: $<0.001)$. Female sex, Medicaid insurance, and hospitalization were associated with significantly higher SPD. Female sex and hospitalization were also associated with significantly higher stress.

\section{Discussion}

In this study, physical health-related quality of life improved and perceived stress decreased at 3 months compared to baseline for adults with positive COVID-19 test results. This relationship was held after adjusting for socio-demographics, comorbidity burden, and severity of disease (hospitalization or ICU stay). Mental healthrelated quality of life and serious psychological distress showed no change over time in this sample. Results suggest that the influence of COVID-19 on physical healthrelated quality of life and general stress may resolve over time, however, the mental health-related quality of life and serious psychological distress, which better captures the influence of mental health on daily activities, work, and social activities may not.

These results add to the growing number of studies that highlight the long-term symptoms and effects of COVID-19 on survivors [21,22]. A recent systematic review and metaanalysis showed the five most common symptoms are fatigue (58\%), headache (44\%), attention disorder (27\%), hair loss (25\%), and dyspnea (24\%) with follow-up time ranging from 15 to 110 days post infection [22]. Only a few studies have assessed the long-term effects on quality of life, psychological distress, and perceived stress. A structured review on the impact of COVID-19 on health-related quality of life found the impact was considerable on quality of life scores, and noted higher impact in acute COVID cases, women, older ages, individuals with more severe disease, and studies in low-income countries [23]. Three studies conducted in China using the SF-36, which provides similar scores as the SF-12 used in this study, found an overall mean ranged from 60.4 to 86.4 [23] Physical functioning scores ranged from 53.2 to 95.1 , and mental health function scores ranged from 61.6 to 84.0 [23] Both baseline and 3-month scores in this study conducted in the US align with the lower end of the range for the studies conducted in China. Studies conducted in Europe using the EQ-5D (EuroQol-5 Dimension) also found differences by country, with overall quality of life scores lower in a study conducted in Belgium than studies conducted in the United Kingdom and Norway [23]. In addition to differences by country, existing studies highlighted differences over time and disease severity. An Italian caseseries with mean follow-up of 60 days showed $44.1 \%$ had worsened quality of life over time [24]. A study from the United Kingdom reporting on post-discharge symptoms assessed 29 to 71 days after discharge showed psychological distress was present in $46.9 \%$ in the ICU group and $23.5 \%$ in ward (i.e., inpatient, non-ICU) group [25]. In this same cohort, there was a clinically significant decrease in quality of life, assessed by EQ-5D, in $68.8 \%$ of the ICU group and $45.6 \%$ of the ward group [25]. Another study from the 


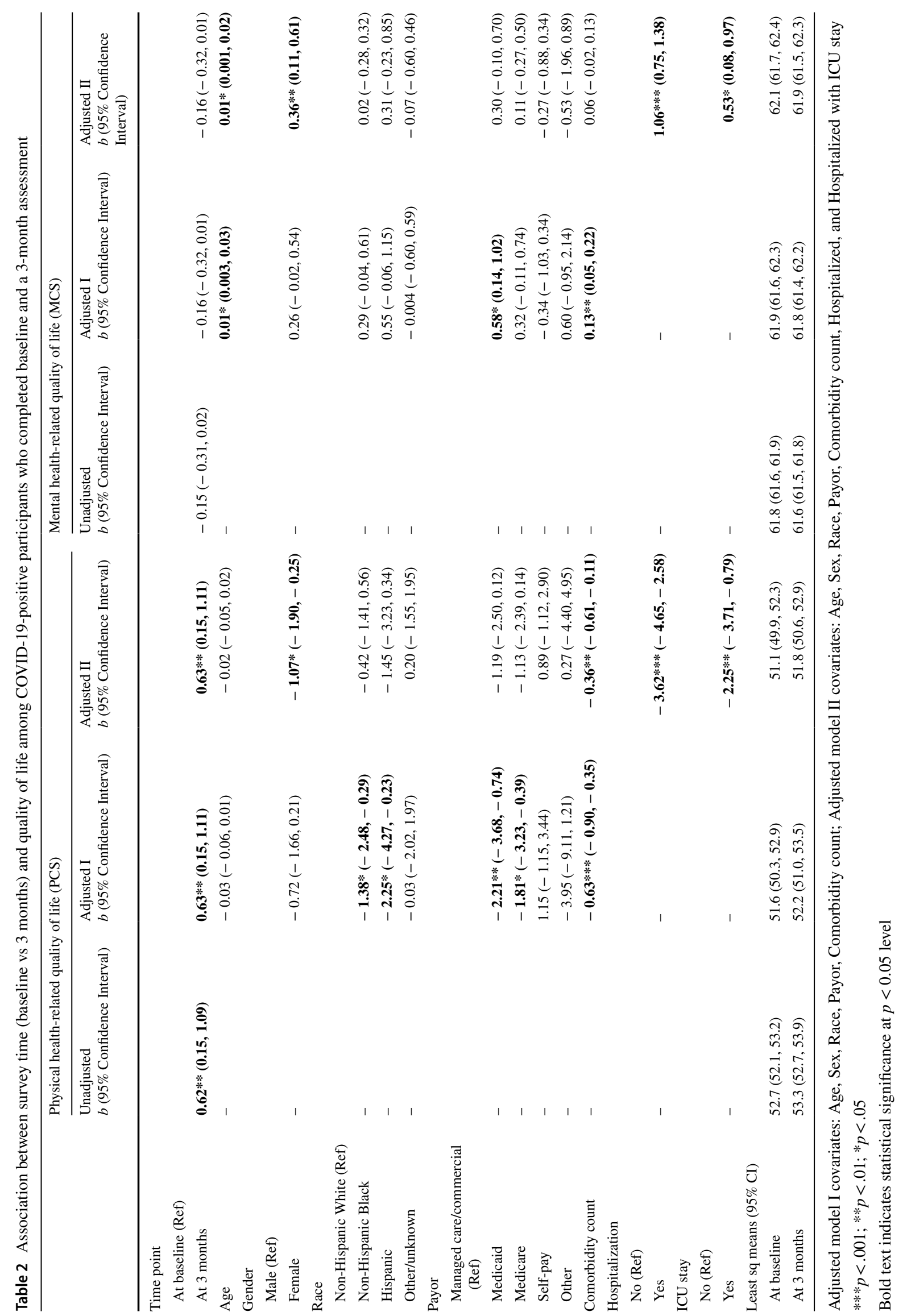




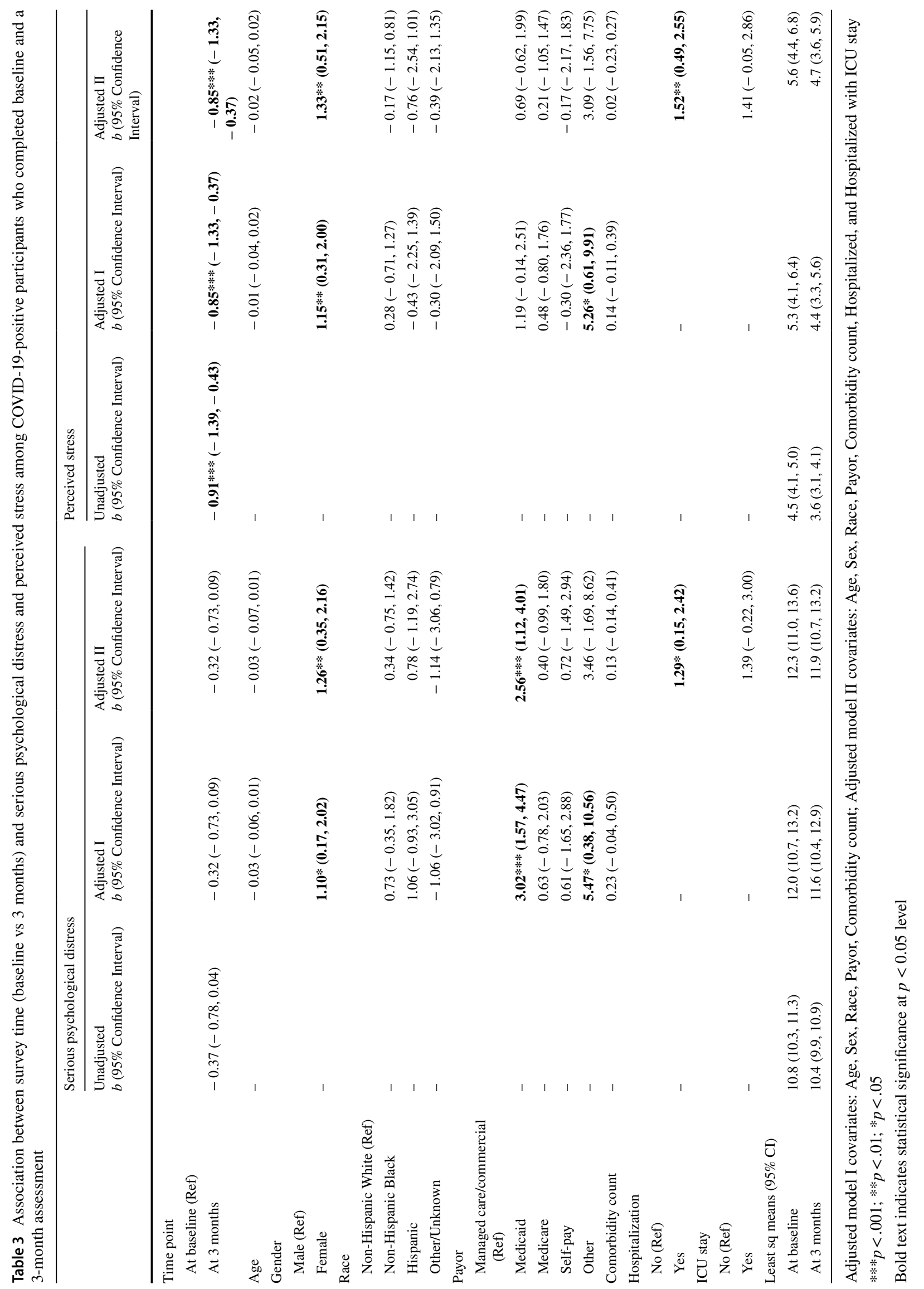


United Kingdom reported similar reduction in quality of life (using the SF-36) on follow-up, with physical healthrelated quality of life significantly lower in the cohort with severe disease, such as ICU admission [26]. Not all studies have noted this difference, however, as a French study with mean follow-up of 110.9 days found quality of life (using the EQ-5D scale) was satisfactory and no difference existed between ICU and ward patients [27].

In contrast to the findings from Europe which showed quality of life outcomes, without adjustment, were worse over time, our study showed improved physical healthrelated quality of life and perceived stress at 3-month follow-up after adjustment for various factors, including severity (ICU and non-ICU admissions). Applicability to the U.S. of findings on quality of life from European studies remains uncertain given the difference in experience of and response to the COVID-19 pandemic [28]. Our study potentially contradicts findings from many studies on adverse long-term outcomes after acute SARS-CoV-2 infection. As such, this study adds to the evolving knowledge on the long-term effects of COVID-19 in terms of quality of life and mental health-related outcomes, such as perceived stress and serious psychological distress, suggesting effects across and between populations need to be continually assessed. Patient characteristics such as female sex, Medicaid insurance, and higher comorbidity count were associated with lower physical health-related quality of life and higher SPD. Thus, clinicians should be vigilant in assessment of long-term outcomes, particularly for vulnerable populations. Clinical severities, such as hospitalization and ICU stay, were also associated with lower physical health-related quality of life, higher SPD, and higher stress, highlighting the need to consider this group for ongoing assessment.

The growing concern for long-term outcomes after COVID-19 infection is based on the previous studies of severe acute respiratory syndrome (SARS) and Middle East respiratory syndrome (MERS) coronavirus infections showing persistent symptoms and psychological impairment. A systematic review and meta-analysis of 28 studies on long-term outcomes on the survivors of SARS and MERS outbreaks found that stress, anxiety, and depression were observed in up to one-third of patients beyond 6 months after discharge from the hospital, and low scores in quality of life (SF-36) were observed even 12 months after discharge [29]. Given this dismal data on the post-discharge outcomes of other groups of coronavirus infections, our study suggests improvement in physical health-related quality of life and perceived stress in COVID-19 survivors, despite the possibility of ongoing clinical symptoms. Longer follow-up will be needed to assess longer-term changes, for example, to determine if improvements in physical health-related quality of life and perceived stress are maintained over time, or if mental health-related quality of life and psychological distress do improve with longer follow-up. These results suggest that dedication of resources and attention to mental health care after COVID-19 is important despite recovery of physical health-related quality of life and longer-term support may be needed.

\section{Limitations}

Our study has a few limitations that should be noted. First, it is based on a single academic health system, which may limit the generalizability of results. Additional studies that continue to follow patients over longer periods of time and in diverse settings are needed to inform clinical care and research. Second, while this study leverages the combined strengths of medical record and survey data, there remain some variables that are not captured, including community level factors that could have an influence on long-term health. Influence on general constructs, such as general stress, may also be more fully understood through collection of longer scales that capture the types and drivers of stress, or future qualitative work allowing individuals to describe the context and experience. Third, long-term clinical symptoms were not captured in this study to differentiate from long COVID syndrome or included in analyses as covariates to adjust relationships. More detailed analysis investigating the interaction between clinical and social factors on long-term outcomes is an important next step. Finally, due to collection of data being initiated after the start of the pandemic, scores for quality of life, stress, and serious psychological distress could not be compared to levels found prior to the pandemic.

\section{Conclusion}

Our survey-based, longitudinal study of adult patients diagnosed with COVID-19 showed improvement in physical health-related quality of life and reduction in perceived stress at 3-month follow-up compared with baseline. Our study adds to the growing body of literature on the longterm effects of COVID-19 and contradicts many studies by showing some improved long-term outcomes. Clinicians should consider assessment of long-term outcomes, particularly for vulnerable populations found to have lower quality of life and higher SPD, including female sex, individuals with Medicaid insurance, individuals with higher number of comorbidities, and those hospitalized or with an ICU stay. Further research with a larger cohort and longer-term follow-up is needed to identify if changes in physical health-related quality of life are maintained over 
time, or if mental health-related quality of life does change with longer time from illness. The current study provides helpful information to clinicians caring for COVID-19 survivors and policy makers to gauge and address the longterm impact of COVID-19 and highlight the importance of taking both physical and mental health-related quality of life changes into account over time.

Supplementary Information The online version contains supplementary material available at https://doi.org/10.1007/s11136-022-03081-7.

Funding Effort for this study was supported by the National Institute of Diabetes and Digestive and Kidney Diseases (Grant K24DK093699, R01DK118038, R01DK120861, Principal Investigator (PI): Leonard Egede, MD), National Institute on Minority Health and Health Disparities (R01MD013826, PI: Egede/Walker), and the American Diabetes Association (1-19-JDF-075, PI: Walker). Funders had no role in study design, data collection, analysis, or interpretation of the results.

Data availability Data may be shared upon contact of corresponding author.

\section{Declarations}

Conflict of interest The authors declare no conflict of interest.

Ethical approval This study was conducted according to the guidelines laid down in the Declaration of Helsinki and all procedures involving human subjects/patients were approved by the Institutional Review Board of the Medical College of Wisconsin, Milwaukee, WI, USA.

\section{References}

1. World Health Organization (WHO). WHO coronavirus (COVID19) dashboard. Retrieved April 19, 2021 from https://covid19. who.int/

2. Wisconsin Department of Health. Wisconsin COVID-19 summary statistics. Retrieved April 19, 2021 from https://www.dhs.wisco nsin.gov/covid-19/data.htm\#summary

3. Egede, L. E., Walker, R. J., Garacci, E., \& Raymond, J. R. (2020). Racial/ethnic differences in COVID-19 screening, hospitalization, and mortality in Southeast Wisconsin. Health Affairs, 39, $1926-1934$.

4. Lopez-Leon, S., Wegman-Ostrosky, T., Perelman, C., Sepulveda, R., Rebolledo, P. A., Cuapio, A., \& Villapol, S. (2021). More than 50 long-term effects of COVID-19: A systematic review and metaanalysis. MedRxiv. https://doi.org/10.1101/2021.01.27.21250617

5. Goërtz, Y. M. J., Van Herck, M., Delbressine, J. M., Vaes, A. W., Meys, R., Machado, F. V. C., Houben-Wilke, S., Burtin, C., Posthuma, R., Franssen, F. M. E., van Loon, N., Hajian, B., Spies, Y., Vijlbrief, H., van 't Hul, A. J., Janssen, D. J. A., \& Spruit, M. A. (2020). Persistent symptoms 3 months after a SARS-CoV-2 infection: The post-COVID-19 syndrome? ERJ Open Research, 6(4), 00542-02020.

6. Lancet, T. (2021). Understanding long COVID: A modern medical challenge. Lancet, 398(10302), 725. https://doi.org/10.1016/ S0140-6736(21)01900-0

7. Carfî, A., Bernabei, R., \& Landi, F. (2020). Gemelli against COVID-19 post-acute care study group. Persistent symptoms in patients after acute COVID-19. JAMA, 324(6), 603-605. https:// doi.org/10.1001/jama.2020.12603

8. Sudre, C. H., Murray, B., Varsavsky, T., Graham, M. S., Penfold, R. S., Bowyer, R. C., Pujol, J. C., Klaser, K., Antonelli, M., Canas, L. S., Molteni, E., Modat, M., Jorge Cardoso, M., May, A., Ganesh, S., Davies, R., Nguyen, L. H., Drew, D. A., Astley, C. M., ... Steves, C. J. (2021). Attributes and predictors of long COVID. Nature Medicine, 27(4), 626-631. https://doi.org/10. 1038/s41591-021-01292-y

9. Garratt, A. M., Ghanima, W., Einvik, G., \& Stavem, K. (2021). Quality of life after COVID-19 without hospitalization: Good overall but reduced in some dimensions. Journal of Infection, 82, 186.

10. Taquet, M., Geddes, J. R., Husain, M., Luciano, S., \& Harrison, P. J. (2021). 6-month neurological and psychiatric outcomes in 236379 survivors of COVID-19: A retrospective cohort study using electronic health records. Lancet Psychiatry, 8, 416.

11. COVID-19 disrupting mental health services in most countries, WHO survey. World Health Organization News Release; 5 October 2020 News release. Retrieved April 19, 2021 from https:// www.who.int/news/item/05-10-2020-covid-19-disrupting-mentalhealth-services-in-most-countries-who-survey

12. Leach, C. R., Rees-Punia, E., Newton, C. C., Chantaprasopsuk, S., Patel, A. V., \& Westmaas, J. L. (2021). Stressors and other pandemic-related predictors of prospective changes in psychological distress. Lancet Regional Health America, 4, 100069.

13. Lamontagne, S. J., Winters, M. F., Pizzagalli, D. A., \& Olmstead, M. C. (2021). Post-acute sequelae of COVID-19: evidence of mood \& cognitive impairment. Brain Behaviour Immunity Health, 17,100347

14. Ware, J. E., Kosinski, M., \& Keller, S. D. (1996). A 12-item shortform health survey: Construction of scales and preliminary tests of reliability and validity. Medical Care, 34, 220-233.

15. Ware, J., Kosinski, M., \& Keller, S. (1995). SF-12: How to score the SF-12 physical and mental summary scales (2nd ed.). The Health Institute, New England Medical Center.

16. Kessler, R. C., Andrews, G., Colpe, L. J., Hiripi, E., Mroczek, D. K., Normand, S. L. T., Walters, E. E., \& Zaslavsky, A. M. (2002). Short screening scales to monitor population prevalence and trends in non-specific psychological distress. Psychological Medicine, 32, 959-976.

17. Umucu, E., Fortuna, K., Jung, H., Bialunska, A., Lee, B., Mangadu, T., Storm, M., Ergun, G., Mozer, D. A., \& Brooks, J. (2021). A national study to assess validity and psychometrics of the short Kessler psychological distress scale (K6). Rehabilitation Counseling Bulletin, 65, 1-10.

18. Cohen, S., \& Williamson, G. (1988). Perceived stress in a probability sample of the United States. In S. Spacapan \& S. Oskamp (Eds.), The social psychology of health. Sage.

19. Quan, H., Sundararajan, V., Halfon, P., Fong, A., Burnand, B., Luthi, J. C., Saunders, L. D., Beck, C. A., Feasby, T. E., \& Ghali, W. A. (2005). Coding algorithms for defining comorbidities in ICD-9-CM and ICD-10 administrative data. Medical Care, 1, $1130-1139$.

20. R Core Team (2020). $R$ : A language and environment for statistical computing. $R$ Foundation for Statistical Computing, Vienna, Austria. https://www.R-project.org/. Accessed 11 Jan 2022.

21. Nalbandian, A., Sehgal, K., Gupta, A., Madhavan, M. V., McGroder, C., Stevens, J. S., et al. (2021). Post-acute COVID-19 syndrome. Nature Medicine, 27(4), 601-615.

22. Lopez-Leon, S., Wegman-Ostrosky, T., Perelman, C., Sepulveda, R., Rebolledo, P. A., Cuapio, A., et al. (2021). More than 50 Long-term effects of COVID-19: A systematic review and metaanalysis. MedRxiv, 32, 1613. https://doi.org/10.1101/2021.01.27. 21250617 
23. Poudel, A., Zhu, S., Cooper, N., Roderick, P., Alwan, N., Tarrant, C., Ziauddeen, N., \& Yao, G. L. (2021). Impact of COVID-19 on health-related quality of life of patients: A structured review. PLoS ONE, 16(10), e0259164.

24. Carfî, A., Bernabei, R., \& Landi, F. (2020). Persistent symptoms in patients after acute COVID-19. JAMA, 324(6), 603-605.

25. Halpin, S. J., McIvor, C., Whyatt, G., Adams, A., Harvey, O., McLean, L., et al. (2021). Postdischarge symptoms and rehabilitation needs in survivors of COVID-19 infection: A cross-sectional evaluation. Journal of Medical Virology, 93(2), 1013-1022.

26. Arnold, D. T., Hamilton, F. W., Milne, A., Morley, A. J., Viner, J., Attwood, M., et al. (2020). Patient outcomes after hospitalisation with COVID-19 and implications for follow-up: Results from a prospective UK cohort. Thorax, 76(4), 399-401.

27. Garrigues, E., Janvier, P., Kherabi, Y., Le Bot, A., Hamon, A., Gouze, H., et al. (2020). Post-discharge persistent symptoms and health-related quality of life after hospitalization for COVID-19. Journal of Infection, 81(6), e4-e6.
28. Melo-Oliveira, M. E., Sá-Caputo, D., Bachur, J. A., Paineiras-Domingos, L. L., Sonza, A., Lacerda, A. C., et al. (2021). Reported quality of life in countries with cases of COVID19: A systematic review. Expert Review of Respiratory Medicine, 15(2), 213-220.

29. Ahmed, H., Patel, K., Greenwood, D. C., Halpin, S., Lewthwaite, P., Salawu, A., et al. (2020). Long-term clinical outcomes in survivors of severe acute respiratory syndrome and Middle East respiratory syndrome coronavirus outbreaks after hospitalisation or ICU admission: A systematic review and meta-analysis. Journal of Rehabilitation Medicine, 52(5), 00063.

Publisher's Note Springer Nature remains neutral with regard to jurisdictional claims in published maps and institutional affiliations. 